\title{
Reversible cardiomyopathy-tachycardiomyopathy in children
}

\author{
Alev Arslan¹, İlkay Erdoğan¹, Birgül Varan¹, Mustafa Yılmaz², Mehmet Bülent Özin², \\ Niyazi Kürşad Tokel ${ }^{1}$ \\ Division of ${ }^{1}$ Pediatric Cardiology, Department of Pediatrics and ${ }^{2}$ Cardiology, Başkent University Faculty of Medicine, \\ Turkey.E-mail:alevkiziltas@gmail.com
}

Received: 11th December 2017, Revised: 25th May 2018, Accepted: 1st September 2018

SUMMARY: Arslan A, Erdoğan İ, Varan B, Yılmaz M, Özin MB, Tokel NK. Reversible cardiomyopathy-tachycardiomyopathy in children. Turk J Pediatr 2019; 61: 552-559.

Tachycardia-induced cardiomyopathy (tachycardiomyopathy) is defined by the presence of a sustained tachycardia that results in left ventricular systolic dysfunction. Restoration of cardiac function is dependent on the control of tachyarrhythmias. We report a series including ten children with tachycardia-induced cardiomyopathy with different etiologies. The medical records of patients with tachycardiomyopathy who were managed in a Pediatric Cardiology Clinic between the years of 2014-2017 were reviewed retrospectively. Ten children ( 3 female, 7 male) were diagnosed with tachycardiomyopathy. The median age of the patients was 12 years (range: 4-15.8). Five had atrial tachycardia, two had ventricular tachycardia, the others had Mahaim fiber tachycardia, permanent junctional reciprocating tachycardia and atrioventricular reentrant tachycardia. Seven patients had catheter ablation and three patients who had previous heart surgery were treated with antiarrhythmic drugs. Median ejection fraction was $33 \%$ (range: 10-48), median left ventricle end-diastolic diameter was $55 \mathrm{~mm}$ (range: 30-78). All showed complete recovery with median ejection fraction $60 \%$ (range: 55 78). Two patient with severe heart failure required extracorporeal membrane oxygenation support, one of them had ventricular assist device support but the device was removed after successful ablation. After two years this patient required permanent pacemaker implantation due to complete atrioventricular block. Tachycardia-induced cardiomyopathy is a rare and treatable cause of heart failure. Early recognition is critical, aggressive treatment aimed at controlling the arrhythmia results in symptom resolution and recovery of ventricular function.

Key words: ablation, cardiomyopathy, extracorporeal membrane oxygenation, tachycardia.

Tachycardia-induced cardiomyopathy, also known as tachycardiomyopathy has been defined as cardiac dysfunction resulting from a high and/or irregular ventricular rate that is completely or partially reversible after normalization of the rhythm abnormality. Persistent tachycardia leads to severe biventricular systolic dysfunction resulting in reduced cardiac output. ${ }^{1}$ Early recognition and prompt treatment of the arrhythmia using pharmacological or ablative techniques results in symptom resolution and recovery of ventricular function. In the largest pediatric series of tachycardiomyopathy, atrial ectopic tachycardia (59\%), permanent junctional reciprocating tachycardia $(23 \%)$ and ventricular tachycardia (7\%) were the most commonly encountered arrhythmias according to Moore et al. ${ }^{2}$ We report a series including ten children with tachycardia-induced cardiomyopathy with different etiologies.

\section{Material and Methods}

Medical records of patients with tachycardiainduced cardiomyopathy who were managed 
in our clinic between the years of 2014-2017 were reviewed retrospectively and those who underwent radiofrequency catheter ablation or cryoablation were identified. Tachycardia induced cardiomyopathy is defined by the presence of a sustained tachycardia (or frequent episodes of tachycardia or very frequent ectopy) that results in left ventricular systolic dysfunction. The diagnosis was confirmed with the improvement of systolic function after normal sinus rhythm was achieved. The study protocol was approved by the Institutional Ethics Committee of our hospital (Project No: KA17/323).

\section{Results}

Ten children ( 3 female, 7 male) were diagnosed as tachycardiomyopathy. Seven of them had no previous medical problems or tachycardia history, but three had prior heart surgery including Senning operation for transposition of great arteries, correction of total anomalous pulmonary venous connection and closure of ventricular septal defect. The median age of the patients was 12 years (range: 4-15.8).

Systolic heart dysfunction was detected in all subjects initially. Median ejection fraction (EF) was $33 \%$ (range: 10-48), median shortening fraction was $16 \%$ (range: 8 - 25), median left ventricle end diastolic diameter was $55 \mathrm{~mm}$ (range: $30-78$ ) on admission. All patients had complete recovery with $60 \%$ (range: $55-78$ ) median EF.

Etiology of tachycardia was atrial tachycardia in five patients, ventricular tachycardia in two patients, permanent junctional reciprocating tachycardia in one patient, atrioventricular reentrant tachycardia in one patient and finally Mahaim tachycardia and atrioventricular nodal reentrant tachycardia concomitantly in one patient.

Treatment: Initially, direct-current cardioversion was performed in three patients and restoration of sinus rhythm was noted in two of them immediately, after amiodarone infusion remained in one patient. Seven patients who had successful radiofrequency catheter ablation had been treated with amiodarone (four patients), sotalol (two patients) and metoprolol (one patient) before the ablation therapy. Three patients who had previous heart surgery were successfully treated with antiarrhythmic drugs; amiodarone (one patient) and sotalol (two patients) (Table I).

Table I. Diagnosis and Management Modalities of the Patients.

\begin{tabular}{|c|c|c|c|c|c|c|c|c|c|c|}
\hline Case & $\begin{array}{l}\text { Age } \\
\text { (year) }\end{array}$ & Arrhythmia & $\mathrm{CV}$ & ECMO & Medication & RFA & $\begin{array}{l}\text { First EF } \\
(\%)\end{array}$ & $\begin{array}{l}\text { Last EF } \\
(\%)\end{array}$ & $\begin{array}{c}\text { Prior } \\
\text { heart } \\
\text { surgery }\end{array}$ & $\begin{array}{l}\text { Follow-up } \\
\text { (month) }\end{array}$ \\
\hline 1 & 11.8 & $\begin{array}{l}\text { Mahaim accessory } \\
\text { pathway }+ \text { AVNRT }\end{array}$ & + & - & Amiodarone & + & 42 & 78 & - & 24 \\
\hline 2 & 14.5 & VT & + & - & Amiodarone & + & 44 & 62 & - & 30 \\
\hline 3 & 13.0 & Atrial tachycardia & - & - & None & + & 17 & 58 & - & 17 \\
\hline 4 & 12.3 & Atrial tachycardia & - & + & Amiodarone & + & 18 & 62 & - & 34 \\
\hline 5 & 14.5 & PJRT & - & + & Sotalol & + & 10 & 55 & - & 13 \\
\hline 6 & 15.8 & VT & + & - & Metoprolol & + & 47 & 68 & - & 27 \\
\hline 7 & 7.9 & AVRT & - & - & Amiodarone & + & 38 & 69 & - & 15 \\
\hline 8 & 7.0 & Atrial tachycardia & - & - & Amiodarone & - & 32 & 60 & $\begin{array}{l}\text { VSD } \\
\text { repair }\end{array}$ & 15 \\
\hline 9 & 4.0 & Atrial tachycardia & - & - & Sotalol & - & 48 & 62 & Senning & 16 \\
\hline 10 & 5.0 & Atrial tachycardia & - & - & Sotalol & - & 48 & 64 & $\begin{array}{l}\text { TAPVC } \\
\text { repair }\end{array}$ & 18 \\
\hline
\end{tabular}

AVNRT: atrioventricular nodal reentry tachycardia, AVRT: atrioventricular reentry tachycardia, CV: cardioversion, ECMO: extracorporeal membrane oxygenation, EF: ejection fraction, RFA: radiofrequency catheter ablation, TAPVC: total anomalous pulmonary venous connection, VSD: ventricular septal defect, VT: ventricular tachycardia 


\section{Cases}

Case 1: This is the case of a 12-year-old girl with abdominal pain, cough and exercise intolerance. Wide QRS tachycardia with superior axis and left branch block was recorded on electrocardiography and no change was noted after adenosine (Fig. 1). Left ventricular ejection fraction was $42 \%$, biatrial enlargement and moderate tricuspid insufficiency was noted on echocardiography. Arrhythmia was terminated by direct-current cardioversion and complete recovery of heart functions were noted at first week. She was maintained with amiodarone for two months and then switched to metoprolol. Wide QRS tachycardia with superior axis and left bundle branch block (cycle length $360 \mathrm{msec}$ ) was provoked in the electrophysiologic study. It was diagnosed as Mahaim type tachycardia according to the standard pacing protocols and mapping methods. During burst pacing from high right atrium another tachycardia with narrow QRS (atrioventricular nodal reentry tachycardia) (cycle length $225 \mathrm{msec}$ ) was provoked. The first cryoablation was performed to typical atrioventricular nodal reentry tachycardia, and then radiofrequency catheter ablation was performed to Mahaim tachycardia.

Case 2: This is the case of a 14-year-old girl who was referred on suspicion of myocarditis.
Moderate mitral insufficiency and left ventricle systolic dysfunction was detected on echocardiography. The electrocardiography demonstrated wide QRS tachycardia, right bundle branch block and left axis (Fig. 2). Hence arrhythmia was not terminated by DC cardioversion and amiodarone infusion was started. After two hours of infusion, rhythm was sinus. She was symptom-free with oral metoprolol for eighteen months, but she had multiple ventricular tachycardia episodes, which responded to intravenous verapamil in the last two months. Metoprolol was switched to verapamil but frequent tachycardia attacks continued. Left posterior fascicular tachycardia was provoked (cycle length $330 \mathrm{msec}$ ) on electrophysiologic studies. After successful radiofrequency catheter ablation she required no antiarrhythmic medication.

Case 3: This is the case of a 13-year-old boy who had severe congestive heart failure (NYHA Class IV). He was referred to our clinic for heart transplantation. EF was $17 \%$. Irregular narrow QRS complexes and different configuration $\mathrm{P}$-waves were recorded on electrocardiography. He was diagnosed atrial tachyardia. After successful radiofrequency catheter ablation from the right atrium, he had no antiarrhythmic medication necessity. Ejection fraction was noted as $56 \%$ after 6 months with better clinical symptoms (NYHA Class I).

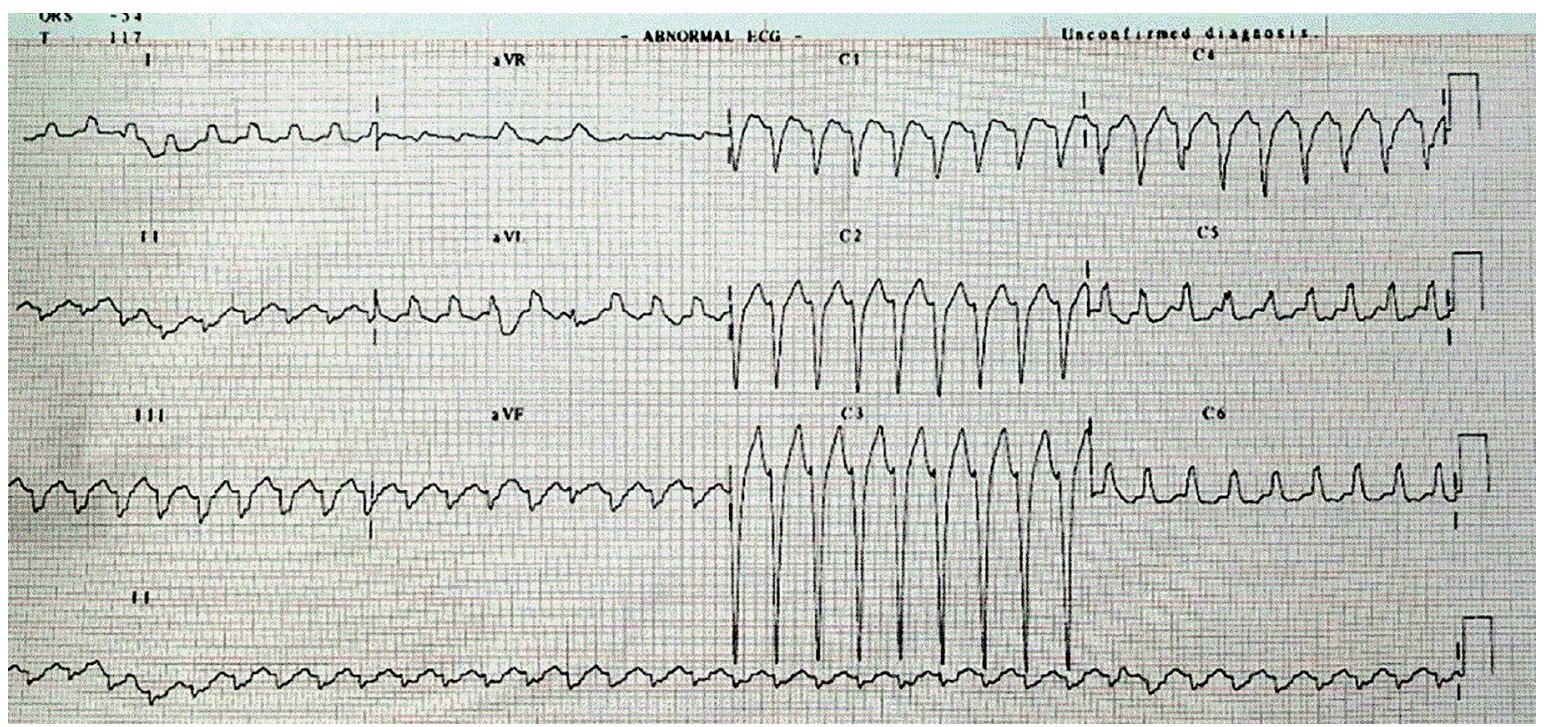

Fig. 1. Case 1: Electrocardiography at time of admission, wide QRS tachycardia with superior axis and left branch block. 


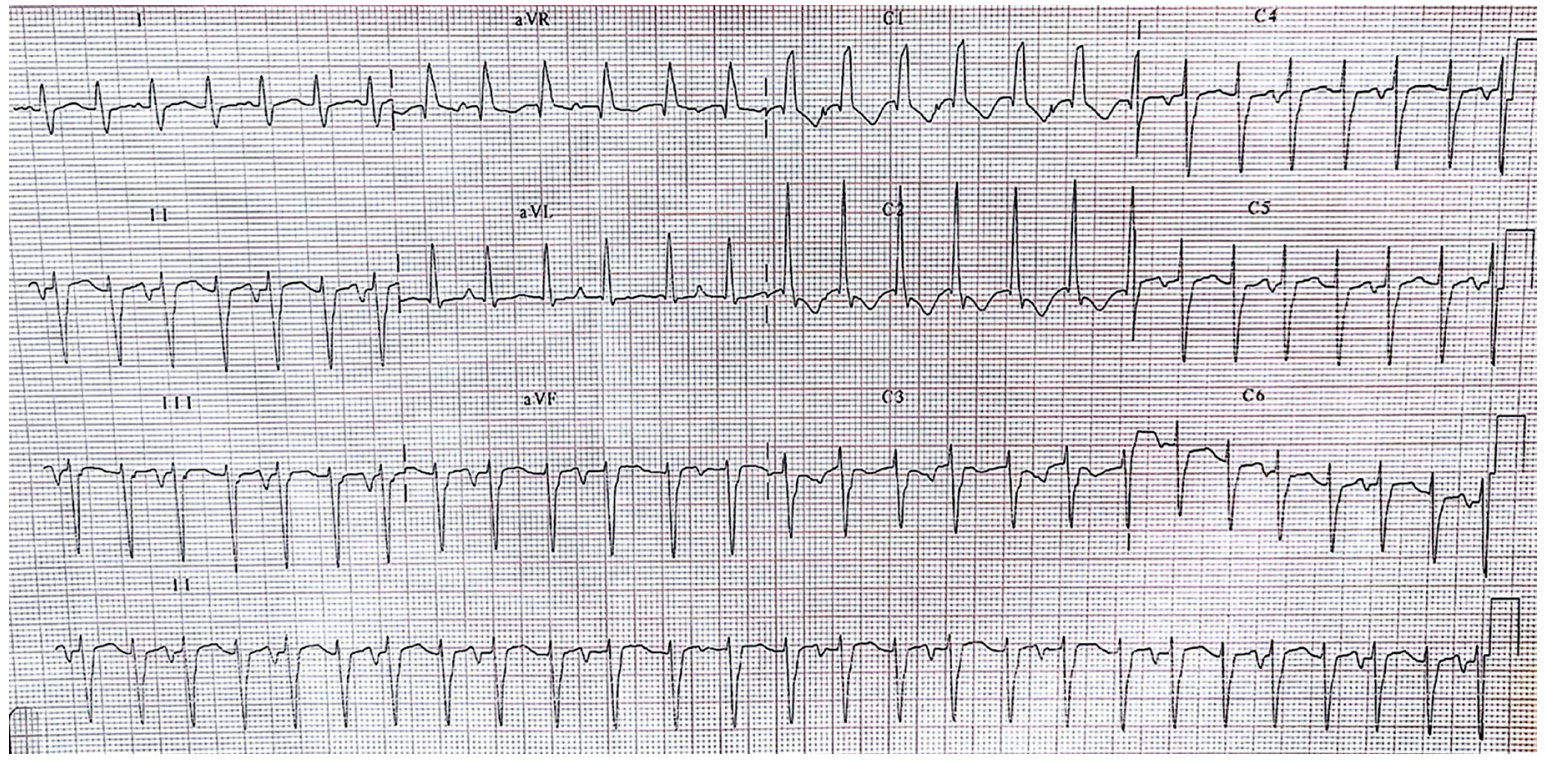

Fig. 2. Case 2: Electrocardiography at time of admission, wide QRS tachycardia, right bundle branch block and left axis.

Case 4: This is the case of a 12-year-old boy who was admitted to the emergency unit due to dyspnea for three days. Left ventricular EF was $18 \%$, severe mitral insufficiency and biventricular dilatation were noted. Narrow QRS tachycardia, negative $\mathrm{P}$ waves in inferior leads and long RP duration were documented on surface electrocardiography and diagnosed as atrial tachycardia (Fig. $3)$. In the course of ablation ventricular fibrillation inadvertently developed and he needed cardiopulmonary resuscitation for a short time. He remained on venoarterial extracorporeal membrane oxygenation for poor ventricular function secondary to the prolonged supraventricular tachycardia. After five days the HeartWare Ventricular Assist Device (HVAD; HeartWare International, Ltd., Framingham, MA) was implanted. Following a month, second radiofrequency

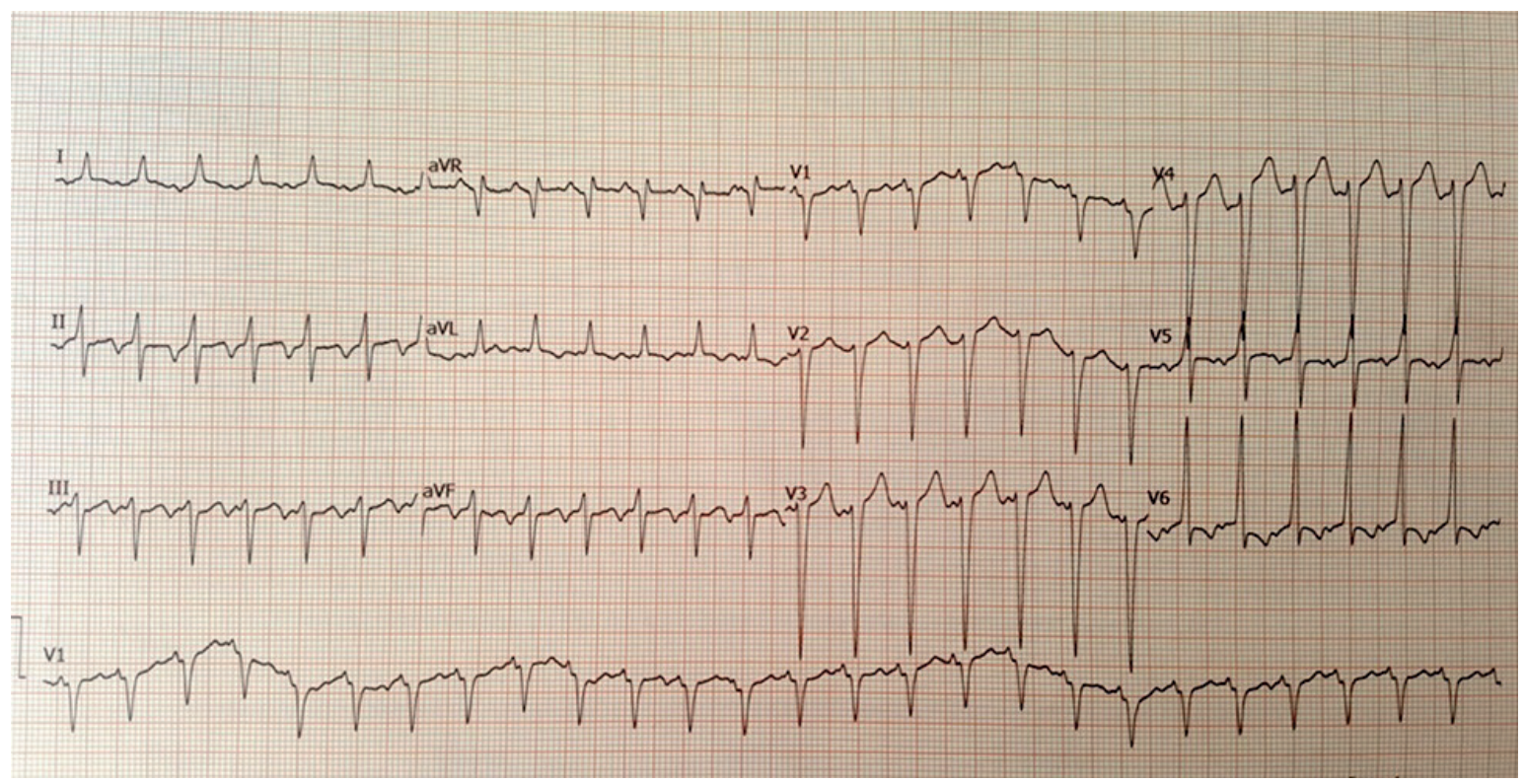

Fig. 3. Case 4: Electrocardiography at time of admission, narrow QRS tachycardia, negative $\mathrm{P}$ waves on inferior leads and long RP duration. 
catheter ablation was successfully applied to atrial tachycardia, which originated from the left anterior side of the interatrial septum. First-degree atrioventricular block (PR duration was $210 \mathrm{msec}$ ) was noted on surface electrocardiography. Systolic heart function improved significantly within a week. Ejection fraction was noted as $56 \%$ after one month and the pump was successfully removed, sewing ring was preserved with the use of a titanium plug (Fittkau Metallbau GmbH, Berlin, Germany). Two years later he had complaints of malaise followed by palpitation. 24-hour Holter electrocardiography monitoring demonstrated the longest pause duration of 2480 milliseconds. After one week he had a syncopal episode for about 5 minutes, an intermittent complete atrioventricular block was recorded. Dual-chamber permanent pacemaker was implanted.

Case 5: This is the case of a 15-year-old boy who was referred on suspicion of myocarditis. Ejection fraction was $10 \%$, left ventricle end diastolic diameter was $79 \mathrm{~mm}$. Narrow QRS tachycardia, and negative $\mathrm{P}$ waves in inferior leads and long RP duration were documented on surface electrocardiography. He was diagnosed as permanent junctional reciprocating tachycardia (cycle length $360 \mathrm{msec}$ ) according to the standard pacing protocols and mapping methods in the electrophysiologic study. No evidence of earlier atrial activation was found during mapping. Ablation was planned from retrograde aortic catheterization in the left posteroseptal region but immediately after left ventricle catheterization ventricular fibrillation developed. $\mathrm{He}$ required multiple defibrillations and intermittent cardiopulmonary resuscitation. The patient developed cardiogenic shock requiring mechanical ventilation, inotropic support with dobutamine and adrenaline. Venoarterial extracorporeal membrane oxygenation was commenced due to worsening cardiogenic shock. After hemodynamic stability, inotropic support was decreased on the following day. Five days later a second attempt of radiofrequency catheter ablation was successful via retrograde aortic catheterization in the left posteroseptal region. Venoarterial extracorporeal membrane oxygenation support was terminated three days after the ablation. Ejection fraction was
$32 \%$ and he was discharged from hospital in ten days (without any neurological sequelae). Ejection fraction was $42 \%$ in the sixth month and $55 \%$ at the end of the first-year follow-up of period.

Case 6: This is the case of a 15-year-old boy who was admitted to the emergency unit due to chest pain, fever and sweating. He complained of upper respiratory infection symptoms for three days but worsened in last three hours. Wide QRS tachycardia with right bundle branch block and left axis was noted. Arrhythmia was terminated by DC cardioversion and complete recovery of heart functions were noted after two days. He was maintained with metoprolol for a month than electrophysiology study and catheter ablation were performed. Left posterior fascicular tachycardia was provoked (cycle length 290 $\mathrm{msec}$ ) on electrophysiologic study. After successful radiofrequency catheter ablation he required no antiarrhythmic medication.

Case 7: This is the case of a 7-year-old boy who was admitted to the emergency unit due to tachycardia. A narrow QRS tachycardia was terminated with Adenosine. He had moderate systolic dysfunction with pulmonary edema. Inotrope support and diuretic infusion were applied for congestive heart failure. Amiodarone was loaded than maintained. Complete recovery of heart functions was determined at the sixth day of admission. Electrophysiology study and catheter ablation were performed. The left lateral concealed accessory pathway was successfully ablated.

The last 3 patients were evaluated due to newly developed unexplained systolic dysfunction during postoperative follow-up. We demonstrated atrial tachycardia at 24hour Holter ECG monitoring in all cases. Two of them were treated with sotalol and one with amiodarone. Complete recovery of heart functions were detected in the first month follow-up.

\section{Discussion}

The clinical presentation in tachycardiomyopathy consists of symptoms of tachycardia and/or heart failure signs. It can be difficult to determine whether an 
arrhythmia is the initiator or consequence of cardiomyopathy in a patient with tachycardia and heart failure. Thus, tachycardiomyopathy raises a "chicken or egg" question. ${ }^{3}$ The critical diagnostic feature of tachycardiomyopathy is the presence of a pathologic tachycardia or persistent arrhythmia in the presence of an otherwise unexplained cardiomyopathy. Many children present with nonspecific symptoms such as dyspnea, palpitation, cough and recent infection history which frequently leads to a misdiagnosis of myocarditis and dilated cardiomyopathy (DCM). Five of our patients were referred to us with initial diagnosis as acute myocarditis and one with DCM for heart transplantation. Therefore, an electrocardiogram (ECG) and Holter ECG monitorization should be performed and evaluated carefully on any patient presenting with new onset dilated cardiomyopathy.

Thetargetoftachycardiomyopathymanagement is to achieve ventricular heart rate control or to restore the sinus rhythm. Options for the restoration of sinus rhythm include electrical cardioversion, antiarrhythmic drugs, and catheter ablation of the arrhythmia. Chronic or recurrent supraventricular tachycardia associated with ventricular dysfunction and recurrent ventricular tachycardia that is associated with hemodynamic compromise are Class I indications for radiofrequency catheter ablation in pediatric patients according to NASPE Expert Consensus Conference. ${ }^{4}$

In our first case with right ventricular tachycardia, arrhythmogenic right ventricular cardiomyopathy could not be ruled out. In the presence of right ventricular tachycardias, once underlying structural right ventricular disease is excluded, the differential diagnosis should include arrhythmogenic right ventricular cardiomyopathy, Mahaim tachycardia and an idiopathic right ventricular outflow tract tachycardia. ${ }^{5}$ Our patient was diagnosed as Mahaim type tachycardia according to electrophysiologic study. Aizawa et al. ${ }^{6}$ have been reported coexistence of Mahaim type accessory pathway with manifest atrioventricular nodal reentry tachycardia before. Both Mahaim tachycardia and atrioventricular nodal reentran tachycardia were ablated successfully in our case.
Permanent junctional reciprocating tachycardia is a potentially lethal arrhythmia and usually refractory to drug therapy, and its persistence over a long period of time may lead to a tachycardia-induced cardiomyopathy. ${ }^{7-8}$ Heart rates are faster and congestive heart failure is more common at younger ages. Remarkable effectiveness of amiodarone and verapamil against permanent junctional reciprocating tachycardia (success rate of more than $80 \%$ ) were reported by Vaksmann et al. ${ }^{9}$ in small children. After adenosine, tachycardia may terminate with AV or VA block, depending on which limb is more sensitive. Usually, prompt reinitiating tachycardia occurs within seconds as the adenosine wears off. The majority of posterior septal accessory pathway potentials are successfully mapped from the right side. When the earliest atrial activation is $\geq 1 \mathrm{~cm}$ inside the coronary sinus, left-sided ablation via a transeptal or retroaortic approach is preferred to radiofrequency application within the coronary sinus according to Bonney et al. ${ }^{10}$

Idiopathic fascicular left ventricular tachycardia typically presents in young adults and the most frequent clinical presentation is paroxysmal episodes of palpitations, dizziness and syncope. Tachycardiomyopathy has been described in $6 \%$ of cases as a result of persistent tachycardia and it is usually reversible after successful ablation according to Ohe et al. ${ }^{11}$ Although most episodes occur at rest, exercise, emotional stress and catecholamine infusion can act as triggers.

Focal atrial tachycardias mostly develop after surgery for congenital heart disease, although spontaneous cases have been reported in children and adolescents. In our study, 5 of 10 patients had atrial tachycardia, and 3 of them had prior cardiac surgery.

On the left side of the interatrial septum, application of energy is less likely to alter atrioventricular conduction, because the distance to the compact atrioventricular node is greater when compared to the right side. ${ }^{12}$ In our case 4 , radiofrequency catheter ablation was performed from the left side of atrial septum. First-degree atrioventricular block was detected immediately after ablation but he experienced syncope episode due to intermittent complete atrioventricular block 
two years later. The patient received a dualchamber pacemaker and did not experience further syncopal episodes.

Postoperative arrhythmias are more commonly atrial reentry or automatic atrial mechanisms. Postoperative atrial tachycardia occurs in up to $40 \%$ of Mustard/Senning patients, $50 \%$ of Fontan patients, and up to $20 \%$ of patients with repaired atrial septal defect, or tetralogy of Fallot, and less frequently with other defects, like ventricular septal defect. ${ }^{13,14}$ Three of our cases with postoperative arrhythmia had prior cardiac surgery including atrial tissue. We successfully treated them with antiarrhythmic drugs.

In tachycardia induced cardiomyopathy, previous small reports describe weeks to months for functional recovery and years for reverse remodeling. The median time of recovery in a larger study was $<2$ months in children. ${ }^{2}$ Failure to recover should instigate a search for factors, such as subclinical arrhythmia recurrence or an underlying cardiomyopathy. Sudden cardiac death has been reported in patients following symptom recovery and left ventricle EF normalization (suggesting a greater risk in patients with severe baseline left ventricle dysfunction). ${ }^{15}$ Close followup is required even after successful ablation because of the tendency for cardiomyopathy if tachycardia recurs. One of our cases who had a history of venoarterial extracorporeal membrane oxygenation support and ventricular assist device with severe baseline left ventricle dysfunction, required permanent pacemaker due to complete atrioventricular block. Two patients with late recovery even after ablation have a close follow-up with echocardiography per three months and 24hour Holter electrocardiography monitoring per six months.

Extracorporeal membrane oxygenation support is an important tool to resuscitate patients suffering from refractory cardiogenic shock in all age groups. Extracorporeal membrane oxygenation provides a hemodynamically stable and safe platform for antiarrhythmic drug therapy and radiofrequency catheter ablation even in sick infants with incessant tachyarrhythmias. ${ }^{16}$ Ventricular assist devices have become standard therapy for patients with advanced heart failure either as a bridge to transplantation or destination therapy. After radiofrequency catheter ablation and heart function improvement, the device could be removed without any complication. ${ }^{17}$

Tachycardia induced cardiomyopathy has a wide range of clinical manifestations, from asymptomatic patients to tachycardia induced cardiomyopathy or to end-stage heart failure. Early recognition is critical, and aggressive treatment aimed at controlling or eliminating the arrhythmia results in symptom resolution and recovery of ventricular function. Recovery seems independent of treatment strategy (ablation vs. medical therapy). However, cellular and extracellular ultrastructural changes can persist and can contribute to a rapid decline in cardiac function with arrhythmia recurrence, as well as confer a risk of sudden cardiac death.

\section{REFERENCES}

1. Fenelon G, Wijns W, Andries E, Brugada P. Tachycardiomyopathy: Mechanisms and clinical applications. Pacing Clin Electrophysiol 1996; 19: 95106.

2. Moore JP, Patel PA, Shannon KM, et al. Predictors of myocardial recovery in pediatric tachycardia-induced cardiomyopathy. Heart Rhythm 2014; 11: 1163-1169.

3. Gopinathannair R, Etheridge SP, Marchlinski FE, Spinale FG, Lakkireddy D, Olshansky B. Arrhythmiainduced cardiomyopathies: Mechanisms, recognition, and management. J Am Coll Cardiol 2015; 66: 17141728

4. Friedman RA, Walsh EP, Silka MJ et al. NASPE Expert Consensus Conference: Radiofrequency catheter ablation in children with and without congenital heart disease. Report of the writing committee. North American Society of Pacing and Electrophysiology. Pacing Clin Electrophysiol 2002; 25: 1000-1017.

5. Corrado D, Basso C, Thiene G. Arrhythmogenic right ventricular cardiomyopathy: Diagnosis, prognosis, and treatment. Heart 2000; 83: 588-595.

6. Aizawa Y, Suzuki K, Chinushi M, Shibata A. AV nodal reentrant tachycardia with a bystander Mahaim fiber. Jpn Heart J 1988; 29: 891-896.

7. Dorostkar PC, Silka MJ, Morady F, Dick M 2nd. Clinical course of persistent junctional reciprocating tachycardia. J Am Coll Cardiol 1999; 33: 366-375.

8. Aykan HH, Karagöz T, Akın A, İrdem A, Özer S, Çeliker A. Results of radiofrequency ablation in children with tachycardia-induced cardiomyopathy. Anadolu Kardiyol Derg 2014; 14: 625-630. 
9. Vaksmann G, D'Hoinne C, Lucet V, et al. Permanent junctional reciprocating tachycardia in children: A multicentre study on clinical profile and outcome. Heart 2006; 92: 101-104.

10. Bonney WJ, Shah MJ. Incessant SVT in children: Ectopic atrial tachycardia and permanent junctional reciprocating tachycardia. Prog Pediatr Cardiol 2013; 35: $33-40$.

11. Ohe T, Aihara N, Kamakura S, Kurita T, Shimizu W, Shimomura K. Long-term outcome of verapamilsensitive sustained left ventricular tachycardia in patients without structural heart disease. J Am Coll Cardiol 1995; 25: 54-58.

12. Frey B, Kreiner G, Gwechenberger M, Gössinger HD. Ablation of atrial tachycardia originating from the vicinity of the atrioventricular node: Significance of mapping both sides of the interatrial septum. J Am Coll Cardiol 2001; 38: 394-400.

13. Deanfield J, Camm J, Macartney F, et al. Arrhythmia and late mortality after Mustard and Senning operation for transposition of the great arteries. J Thorac Cardiovasc Surg 1988; 96: 569-576.
14. Gewillig M, Wyse RK, de Leval MR, J E Deanfield Early and late arrhythmias and the Fontan operation: Predisposing factors and clinical consequences. $\mathrm{Br}$ Heart J 1992; 67: 72-79.

15. Nerheim P, Birger-Botkin S, Piracha L, Olshansky B. Heart failure and sudden death in patients with tachycardia-induced cardiomyopathy and recurrent tachycardia. Circulation 2004; 110: 247-252.

16. Shebani SO, Ng GA, Stafford P, Duke C. Radiofrequency ablation on veno-arterial extracorporeal life support in treatment of very sick infants with incessant tachymyopathy. Europace 2015; 17: 622-627.

17. Gultekin B, Ersoy O, Ozkan M, Akkaya I, Umaroglu S, Sezgin A. Left ventricular assist device results: Single center experience. Exp Clin Transplant 2015; 13(Suppl 3): 149-152. 